deal with "life demands."

The two major treatment modalities for anxiety-based conditions are medical (physiological) and psychological. Medical treatment, consisting of pharmaceutical products such as antidepressants and anxiolytics, does not address the problem directly, but concentrates on the reduction of anxiety and affective symptoms. This case study focuses on a behavioral intervention. Most clients suffering from PTSD and anxiety-related disorders have an imbalance of the autonomic nervous response, with an increased function of the sympathetic nervous system, and a decreased function of the parasympathetic nervous system. Respiration sinus arrhythmia (RSA) is a noninvasive measure of parasympathetic tone that has been related to emotion regulatory capacity. RSA refers to the cyclical variation in heart rate associated with respiration, which can be modified by rate and depth of breathing. High RSA levels among depressed or anxious individuals predict more pernicious course of illness and a poor prognosis of recovery than lower RSA levels.

Modern signal processing such as Bioview Series IV provides a means of measuring and analyzing heart rate and RSA, so to permit a quantitative, noninvasive method of assessing the autonomic nervous system's effect on the body.

This case study demonstrates the effects of respirationbased training as a modality to increase RSA, and therefore, the influence on the activation of the parasympathetic nervous system.

Keywords: anxiety-related disorders; illness, course of; parasympathetic tone; post-traumatic stress disorder (PTSD); respiratory sinus arrhythmia (RSA); training, respiratory-based; treatment

Prehosp Disast Med 2002;17(s2):s75-76.

\section{Comparison of Mainz Emergency Evaluation System (MEES) and Revised Trauma Score (RTS) for Prediction of Mortality of Trauma Patients \\ S. Grmec; ${ }^{1}$ T. Schaubach, ${ }^{1}$ E. Stok, ${ }^{2}$ J. Ferk ${ }^{3}$ \\ 1. Center for Emergency Medicine, Maribor, Slovenia \\ 2. Ministry for Health, Emergency Medicine Project, Ljubljana, Slovenia \\ 3. Teaching Hospital, Maribor, Slovenia}

Introduction: There are numerous prehospital, descriptive scoring systems, and it is uncertain whether they are efficient in assessing of the severity of injury, and whether they have a prognostic role in the estimation of the injury outcome. The purpose of this study was to assess the value of the Mainz Emergency Evaluation System (MEES) and the Revised Trauma Score (RTS) in predicting the outcome of trauma patients.

Method: In a prehospital setting, values for MEES and RTS were measured for each trauma patient. This study was undertaken over four years (January 1998 to July 2002) and included 286 consecutive patients (188 male and 98 female) hospitalized for trauma (polytrauma, multitrauma, trauma with shock or/and coma). Their ages ranged from 16 to 81 years $(45.3 \pm 169)$. Patients younger than 16 years of age were not included. Sensitivity, specificity, and correct prediction of outcome were assessed using the two severity scores. The best cut-off point in each scoring system was determined using the Youden index. The difference in Youden index was calculated using the $\mathrm{Z}$-score. For each score, the receiver operating characteristic (ROC) curve was obtained. The difference in ROC was calculated using the $Z$-score. A $p$-value of $<0.05$ was considered statistically significant.

Results: For prediction of mortality, the best cut-off points were 18 for MEES and 7 for RTS. The best cut-offs for the Youden index were 0.63 for MEES and 0.60 for RTS. The correct prediction of outcome was achieved in $79.6 \%$ for MEES and $75.8 \%$ for RTS. The area under the ROC curve was $0.83 \pm 0.04$ for MEES and $0.79 \pm 0.06$ for RTS. There were no statistically significant differences among MEES and RTS in terms of correct prediction of outcome, Youden index, or area under ROC curve.

Conclusions: Use of the MEES for prediction of mortality in patients with trauma is equal to use of the RTS, and in a prehospital setting, it is not necessary to combine it with the RTS. The MEES also is valuable in predicting outcome for patients with other diseases. Because of this, the MEES provides a good scoring system for quick evaluation of the emergency status as well as for predicting the outcome of patients in a prehospital setting, especially in mass casualty situations, where the combination of trauma and other diseases is expected (e.g., combination of trauma and poisoning).

Keywords: Mainz Emergency Evaluation System (MEES); mortality; outcome; prediction; prognosis; Revised Trauma Score (RTS); trauma; Youden index Prebosp Disast Med 2002;17(s2):s76.

\section{Characteristics of Biological Terrorism and Managing Measures \\ Prof. Sbusen Guo}

Medical Service Department of the Logistics Command

Academy of Chinese People's Liberation Army, Beijing, China

Since the events of 11 September 2001, more and more attention has been paid to biological terrorism. Characteristics of biological terrorism include: (1) Potentiality; there are more than 1,500 bacteria banks in the world, and an uncountable number of institutes from which terrorists could get biological agents. (2) There have been scattered occurrences, not only in an area, but also in time. (3) Concealed easily, biological agents can be put into food and other materials. (4) Suddenness of appearance make it difficult to forecast. (5) Often, it occurs with another kind of terrorism. (6) Easily hidden with other materials to create panic.

Before an incident occurs, we should prepare regulations and programs, study technology and skills, store up materials, and train personnel. After the incident occurs, we should identify the danger, identify the biological agent, forecast the diseases that may result, evaluate the number of patients and areas into which it is likely to spread, put forward measures to reduce the harm, prevent people from exposure, treat patients, and assess the effectiveness of managing measures and adjust them properly.

Keywords: bacteria banks; characteristics; forecast; prediction; preparation; regulations; responses; terrorism, biological Prebosp Disast Med 2002;17(s2):s76. 